\section{Choroidal circulation in diabetic patients}

\begin{abstract}
Purpose To evaluate choroidal circulatory changes in diabetic patients with and without background diabetic retinopathy (BDR) by measuring the retrobulbar circulation with colour Doppler imaging (CDI).

Methods CDI was used to measure circulatory parameters of the PCA (posterior ciliary artery), CRA (central retinal artery), OA (ophthalmic artery) and the respective veins in 73 diabetic patients and 22 controls in a sitting posture. Among the diabetic patients, 38 patients were without diabetic retinopathy (NDR) and 35 had BDR. A non-parametric Kruskal-Wallis test with a Dunn correction was used for data analysis.

Results End-diastolic velocity (EDV) in the PCA was decreased $(2.55 \pm 0.80 \mathrm{~cm} / \mathrm{s})$ and resistivity index (RI) in the PCA was increased $(0.70 \pm 0.08)$ in BDR patients compared with the control patients' $\operatorname{EDV}(3.23 \pm 1.08 \mathrm{~cm} / \mathrm{s}$, $p=0.01)$ and $\mathrm{RI}(0.62 \pm 0.06, p=0.0003)$. RI in the CRA was significantly higher in the BDR group $(0.74 \pm 0.09)$ than in the control group $(0.68 \pm 0.08, p=0.006)$. RI in the OA was significantly higher in the BDR group $(0.87 \pm 0.06)$ compared both with the NDR group $(0.83 \pm 0.07)$ and with the control group $(0.81 \pm 0.06 ; p=0.007, p=0.004)$. NDR patients had a significantly higher RI in the PCA $(0.67 \pm 0.08)$ than control patients $(0.62 \pm 0.06$, $p=0.01$, while the CRA RI $(0.71 \pm 0.09)$ did not show significant differences from the control group $(0.69 \pm 0.08, p=0.32)$. Decreased EDV in the CRA was detected in NDR patients

$(2.16 \pm 0.76 \mathrm{~cm} / \mathrm{s})$ compared with the controls $(2.72 \pm 0.92 \mathrm{~cm} / \mathrm{s}, p=0.007)$.

Conclusion The results from this study suggest that not only the retinal but also the choroidal circulation is affected in NDR and BDR patients.
\end{abstract}

Key words Choroidal circulation, Colour Doppler imaging, Diabetic retinopathy, Posterior ciliary artery

Histological studies have found diabetic changes in the retina in its outer layers that are known to be dependent on the choroidal circulation. ${ }^{1}$ Choroidal changes which suggest the presence of diabetic choroidopathy have also been described. ${ }^{2,3}$ These changes consist of blood vessel lumen narrowing, ${ }^{2,3}$ capillary dropout and focal scarring. ${ }^{3}$ However, probably
GALINA DIMITROVA, SATOSHI KATO, YASUHIRO TAMAKI, HIDETOSHI YAMASHITA, MIYUKI NAGAHARA, MASAHIKO SAKURAI, SHIGEHIKO KITANO, HARUMI FUKUSHIMA

because it is difficult to assess, choroidal blood flow has been investigated less than retinal blood flow.

The quantitative evaluation of choroidal blood flow changes has mostly been done indirectly, by measuring pulsatile blood flow. Studies investigating pulsatile blood flow found decreased pulsatile blood flow in non-diabetic retinopathy (NDR) patients ${ }^{4}$ and increased pulsatile blood flow in background diabetic retinopathy (BDR) and proliferative diabetic retinopathy (PDR) patients ${ }^{5}$ using a modified pneumotonometer. Unchanged ocular pulse amplitude in NDR patients ${ }^{6}$ and a decreased pulsatile blood flow in BDR and PDR patients ${ }^{7}$ was found using the Langham pneumatic probe. Fundus pulsation measurements did not detect any significant changes in the choroidal circulation in the non-PDR stages of diabetic retinopathy. ${ }^{8}$ Colour Doppler imaging (CDI) studies, which measured posterior ciliary artery blood flow parameters in supine patients, did not find any significant haemodynamic changes in BDR patients. ${ }^{9,10}$

The aim of this study was to investigate the posterior ciliary artery circulation in NDR and BDR patients assuming a sitting posture and using CDI. Previous CDI studies on diabetic patients have measured eye circulation in supine position. ${ }^{9,10}$ However, in this study we investigated the patients while seated, because posture is known to affect the choroidal blood flow $^{11}$ and the erect position has been reported to have a higher reproducibility than the supine position with the CDI method. ${ }^{12}$

Although pulsatile methods are usually used for choroidal blood flow evaluation, in this study we chose the CDI method. The reason was our interest in the quantitative analysis of other circulatory parameters (peak-systolic velocity, end-diastolic velocity, mean velocity, resistivity index) that could be linked to the haemo-rheological and vascular diabetic abnormalities and are different from the parameters estimated by the pulsatile method. Furthermore, we were interested to observe the changes in these parameters with respect to the retinal and ophthalmic circulatory changes, and this would not be possible with the other methods. 
Table 1. Clinical characteristics of control and diabetic patients

\begin{tabular}{lcc}
\hline & Control group & Diabetic group \\
\hline No. & 22 & 73 \\
Age (years) & $62.0 \pm 7.7$ & $62.3 \pm 10.1$ \\
Sex $(\mathrm{F} / \mathrm{M})$ & $11 / 11$ & $31 / 42$ \\
Body mass index $\left(\mathrm{kg} / \mathrm{m}^{2}\right)$ & $22.2 \pm 2.7$ & $23.4 \pm 3.4$ \\
IOP (mmHg) & $14.5 \pm 1.9$ & $15.5 \pm 2.2$ \\
Systolic BP (mmHg) & $128.4 \pm 15.7$ & $130.5 \pm 13.0$ \\
Diastolic BP (mmHg) & $77.7 \pm 8.6$ & $75.3 \pm 8.8$ \\
Pulse rate (beats/min) & $79.7 \pm 13.8$ & $78.5 \pm 12.17$ \\
\hline
\end{tabular}

$\mathrm{IOP}$, intraocular pressure; $\mathrm{BP}$, blood pressure.

\section{Subjects and methods}

\section{Subjects}

A total of 73 diabetes mellitus (DM) patients and 22 control individuals were enrolled into the study (Table 1). The group of DM patients was subdivided into 38 patients with NDR and 35 patients with BDR (Table 2). Data were obtained on only one eye, usually the right eye, unless there was a complication or if the quality of the measurement was not satisfactory. Eyes with photocoagulation and eyes with a history of other ocular diseases except for cataracts were excluded from the study. In some patients there were circulatory parameters that it was not possible to evaluate because the pulse wave was not detectable. We could not measure the central retinal artery in 1 diabetic patient, the posterior ciliary artery in 1 control subject and 1 diabetic patient and the ophthalmic artery in 4 control subjects and 10 diabetic patients. There were 12 hypertensive patients in the NDR group and 13 hypertensive patients in the BDR group. However, patients with a systolic blood pressure (BP) over $150 \mathrm{mmHg}$ were excluded from the study, and we detected no significant differences in $\mathrm{BP}$, pulse rate and intraocular pressure (IOP) between the study groups.

The patients were randomly chosen from those who came for regular check-ups in the diabetic retinopathy outpatient and the general outpatient clinic of the University of Tokyo.

We picked age-matched control subjects from patients who had cataracts and no other ocular diseases or from patients who had one healthy eye and only minor ocular disease in the other eye. In the latter case, the healthy eye was used in our study. There were 5 hypertensive patients in the control group.

Table 2. Clinical characteristics of non-diabetic retinopathy (NDR) and background diabetic retinopathy (BDR) patients

\begin{tabular}{lcc}
\hline & NDR group & BDR group \\
\hline No. & 38 & 35 \\
Type of diabetes & Type 1: 2 & Type 1: 5 \\
& Type 2: 36 & Type 2: 30 \\
Diabetes duration (years) & $9.0 \pm 7.2$ & $18.9 \pm 11.2$ \\
$\mathrm{FBS}(\mathrm{mg} / \mathrm{dl})$ & $155.6 \pm 43.3$ & $153.7 \pm 53.9$ \\
$\mathrm{Hb}$ Alc $(\%)$ & $7.1 \pm 1.1$ & $7.2 \pm 1.0$ \\
Type of treatment & Diet: 11 & Diet: 4 \\
& Drugs: 24 & Drugs: 23 \\
& Insulin: 3 & Insulin: 8 \\
\hline
\end{tabular}

All the patients were informed about the nature of the study and gave their consent to be included. The study was carried out in accordance with the standards of the ethics committee of University of Tokyo School of Medicine.

\section{Measurements of ocular blood flow}

All the measurements were performed with a Powervision SSA-380 A (Toshiba, Tokyo, Japan) using a $7 \mathrm{MHz}$ transducer. One observer did all the measurements (G.D.).

The patients were measured while in a sitting position. Patients fixed their gaze about $15 \mathrm{~cm}$ in front of their face and the transducer coupled with sterile ophthalmic gel was applied to their upper lids. The central retinal artery and central retinal vein were found 10-15 mm away from the optic disc, inside the optic nerve. Because the velocity of blood flow in the central retinal artery over the lamina cribosa is usually higher than normal, the gate of the receiver was placed about $5 \mathrm{~mm}$ away from it. The posterior ciliary artery and vein were found parallel to the optic nerve at about the same distance from the fundus as the central retinal artery and vein. The ophthalmic artery and vein were usually found lateral to the optic nerve.

The following haemodynamic parameters were measured in the above-mentioned blood vessels: peak systolic blood velocity (PSV), end-diastolic blood velocity (EDV), mean blood velocity (mean $\mathrm{V}$ ) and resistivity index (RI),

$$
\mathrm{RI}=(\mathrm{PSV}-\mathrm{EDV}) / \mathrm{PSV}
$$

The CDI method allows for accurate RI measurement because its values do not depend on the Doppler angle. We attempted to have the Doppler angle as parallel as possible to the measured blood vessel in order to get more accurate data for blood velocity. Ocular blood flow towards the transducer was depicted as red, away from the transducer as blue.

\section{Statistical analysis}

Because the results of the blood flow parameters did not always follow the normal distribution, a non-parametric Kruskal-Wallis test was used to determine differences between groups. The Dunn correction was applied to test for significant differences among groups. For the data on the patients' clinical characteristics, ANOVA was used. A $p$ value less than 0.05 was regarded as statistically significant.

\section{Results}

\section{Posterior ciliary artery and vein (Table 3)}

EDV in the posterior ciliary artery was significantly lower in the BDR group than in the control group $(p=0.01)$. 
Table 3. Posterior ciliary artery and vein circulatory parameters $(\mathrm{cm} / \mathrm{s})$

\begin{tabular}{cllll}
\hline & PCA PSV & PCA EDV & PCA mean V & PCV mean V \\
\hline Control & $8.54 \pm 2.88$ & $3.23 \pm 1.08$ & $5.44 \pm 1.89$ & $2.37 \pm 0.22$ \\
$(n)$ & $(21)$ & $(21)$ & $(21)$ & $(21)$ \\
NDR & $8.98 \pm 3.35$ & $2.84 \pm 0.99$ & $5.38 \pm 1.87$ & $2.29 \pm 0.26$ \\
$(n)$ & $(38)$ & $(38)$ & $(38)$ & $(38)$ \\
BDR & $8.55 \pm 2.09$ & $2.55 \pm 0.80$ & $4.92 \pm 1.26$ & $2.27 \pm 0.22$ \\
$(n)$ & $(34)$ & $(34) \#$ & $(34)$ & $(34)$ \\
\hline
\end{tabular}

PCA PSV, posterior ciliary artery peak systolic velocity; PCA EDV, posterior ciliary artery end-diastolic velocity; PCV mean V, posterior ciliary vein mean velocity; PCA mean $V$, posterior ciliary artery mean velocity; NDR, non-diabetic retinopathy; BDR, background diabetic retinopathy; $n$, number of eyes.

\#Statistically significant difference from the control group $(p=0.01)$ (Kruskal-Wallis).

RI in the posterior ciliary artery was significantly higher in the NDR $(0.67 \pm 0.08)$ and BDR $(0.70 \pm 0.08)$ groups than in the control group $(0.62 \pm 0.06)(p=0.01$, $p=0.0003$ ) (Fig. 1).

\section{Central retinal artery and vein (Table 4)}

Table 4 shows the data concerning the central retinal artery blood flow parameters. The data show that the lowest values of central retinal artery blood velocity were in the BDR group. The PSV, EDV and mean V values in the central retinal artery were significantly lower in the BDR group compared with the control and NDR groups $(p<0.0001, p=0.009 ; p<0.0001 ; p=0.003 ; p<0.0001$, $p=0.002$ ). NDR patients had significantly lower EDV in the central retinal artery compared with the controls $(p=0.007)$. Central retinal vein mean $\mathrm{V}$ was significantly higher in the BDR than in the NDR group $(p=0.0007)$.

$\mathrm{RI}$ in the central retinal artery was highest in the BDR group. It was significantly higher in the central retinal artery in the BDR group $(0.74 \pm 0.9)$ compared with the control group $(0.68 \pm 0.08)(p=0.006)$ (Fig. 1$)$. RI in the central retinal vein in the BDR group $(0.39 \pm 0.10)$ was significantly higher than in the control $(0.26 \pm 0.11)$ and NDR groups $(0.29 \pm 0.14)(p=0.0002, p=0.0004)$

(Fig. 1).

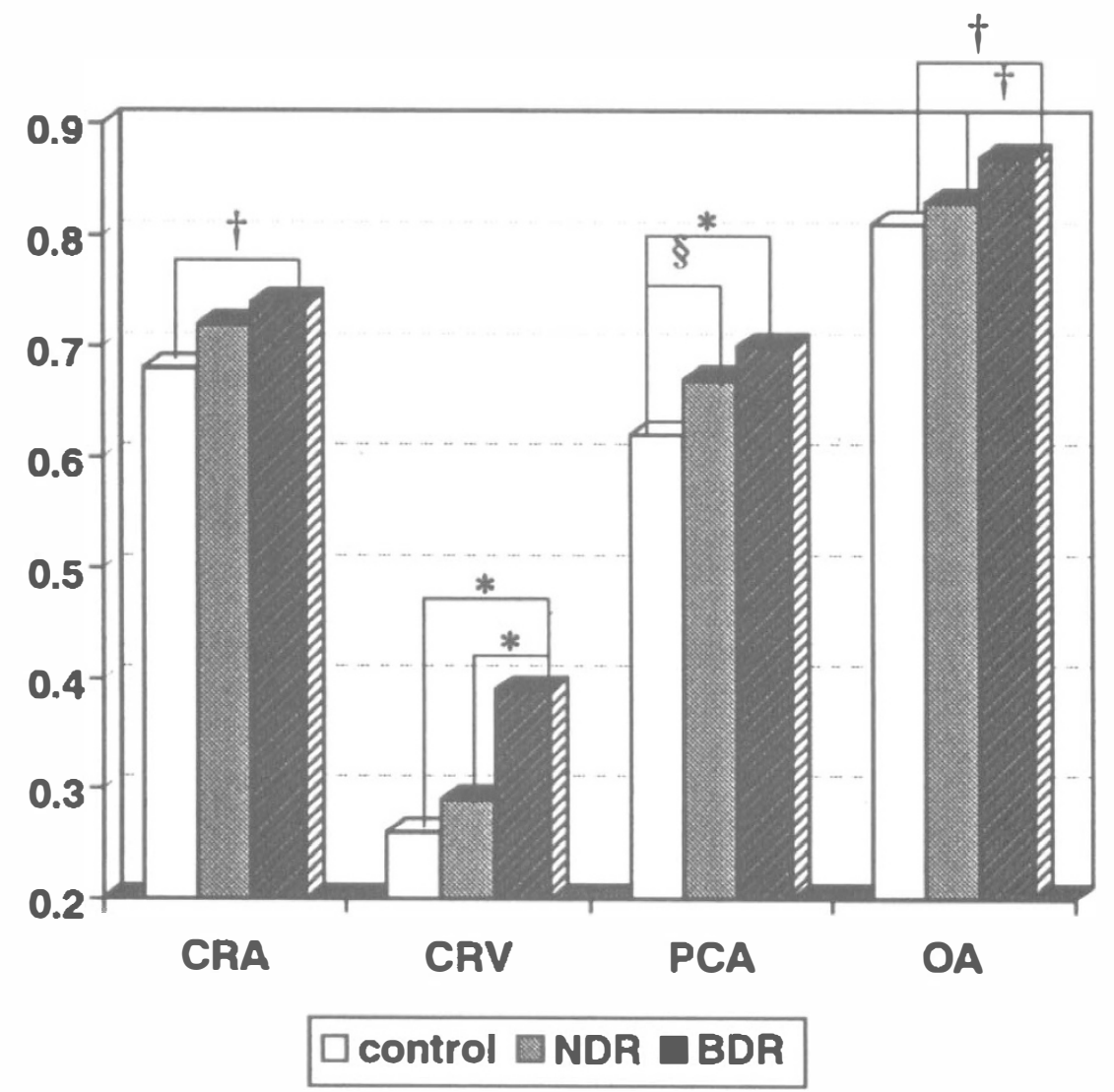

Fig. 1. Resistivity index of the central retinal artery and vein, posterior ciliary artery and ophthalmic artery. CRA, central retinal artery; $C R V$, central retinal vein; PCA, posterior ciliary artery; $O A$, ophthalmic artery; NDR, non-diabetic retinopathy; BDR, background diabetic retinopathy. ${ }^{*} \mathrm{p}=0.0002-0.0004 ; \mathrm{\dagger} \mathrm{p}=0.004-0.007 ; \S \mathrm{p}=0.01$. 
Table 4. Central retinal artery and vein circulatory parameters $(\mathrm{cm} / \mathrm{s})$

\begin{tabular}{cllll}
\hline & CRA PSV & CRA EDV & CRA mean V & CRV mean V \\
\hline Control & $8.56 \pm 2.44$ & $2.72 \pm 0.92$ & $5.05 \pm 1.61$ & $3.64 \pm 0.82$ \\
$(n)$ & $(22)$ & $(22)$ & $(22)$ & $(22)$ \\
NDR & $7.57 \pm 1.79$ & $2.16 \pm 0.76$ & $4.30 \pm 1.17$ & $3.43 \pm 0.66$ \\
$(n)$ & $(38)$ & $(38) \dagger$ & $(37)$ & $(38)$ \\
BDR & $6.36 \pm 1.66$ & $1.61 \pm 0.60$ & $3.36 \pm 0.97$ & $4.24 \pm 1.30$ \\
$(n)$ & $(34)^{*}$ & $(3.4)^{*}$ & $(33)^{*}$ & $(33)^{*}$ \\
\hline
\end{tabular}

CRA PSV, central retinal artery peak systolic velocity; CRA EDV, central retinal artery end-diastolic velocity; CRA mean V, central retinal artery mean velocity; CRV mean $\mathrm{V}$, central retinal vein mean velocity; NDR, non-diabetic retinopathy; BDR, background diabetic retinopathy; $n$, number of eyes.

*Statistically significant difference from the NDR group ( $p=0.009-0.0007)$.

\#Statistically significant difference from the control group $(p<0.0001)$.

†Statistically significant difference from the control group $(p=0.007)$ (Kruskal-Wallis).

\section{Ophthalmic artery and vein (Table 5)}

EDV in the ophthalmic artery was significantly lower in the BDR group compared with the control and NDR groups $(p=0.003 ; p=0.0002)$. Mean $\mathrm{V}$ in the ophthalmic artery was significantly lower in the BDR than the NDR group $(p=0.01)$.

RI in the ophthalmic artery was significantly higher in the BDR group $(0.87 \pm 0.06)$ compared with the control $(0.81 \pm 0.06)$ and NDR groups $(.83 \pm 0.07)(p=0.004$, $p=0.007)$ (Fig. 1).

As shown in Fig. 1, the RI had higher values in all the measured arteries and the central retinal vein in the BDR group compared with the other two groups.

\section{Discussion}

The results from this study that show decreased EDV and increased RI in the posterior ciliary artery support the hypothesis that the choroidal circulation is affected in the early stages of diabetic retinopathy.

The short and long posterior ciliary arteries are responsible for the blood perfusion of the choroid and the optic nerve disc. The pathohistological changes of the choroid in diabetes (basement membrane thickening, narrowing of the capillary lumen, capillary dropout and focal scarring) which were found in electron microscopic ${ }^{2,3}$ studies are concordant with the present finding of decreased EDV and increased RI in the posterior ciliary artery. Because the choroid is responsible for the nourishment of the outer retinal layers and the macula, a circulatory change in the choroidal vessels could be expected to be involved in the pathophysiology of these segments. It has been suggested that activation of the pigment epithelium as might occur with disruption of Bruch's membrane or ischaemia as a result of the loss of the choroidal circulation, would lead to upregulation of vascular endothelial growth factor (VEGF) synthesis and to a directed neovascular response. ${ }^{13}$ This suggests that a change in the choroidal circulation may trigger the proliferative phase.

Studies which measured choroidal blood flow by means of pulsatile ocular blood flow have found decreased pulsatile blood flow in $\mathrm{NDR}^{4,7}$ and BDR patients, ${ }^{7}$ and increased pulsatile blood flow in all grades of DR. ${ }^{5}$ Another study, ${ }^{6}$ using the pulsatile method, did not detect any changes in the choroidal circulation of NDR, BDR or PDR patients. However, the latter study, unlike those previously mentioned, measured young, type 1 diabetes patients. The differences in methods of pulsatile blood flow measurement, age groups, as well as patients' posture, could explain the differences in the results of these studies.

The results in the present study are in agreement with those in previous studies ${ }^{4,5,7}$ using the pulsatile method that have detected a choroidal circulatory alteration in the NDR and BDR stages. If we consider that in 'in vivo' and 'in vitro' experiments a good association has been found between blood flow and blood flow velocity, ${ }^{14}$ then our findings would be closer to the pulsatile method studies that suggested decreased choroidal blood flow in $\mathrm{NDR}^{4,7}$ and BDR patients. ${ }^{7}$ However, without the vessel

Table 5. Ophthalmic artery and vein circulatory parameters $(\mathrm{cm} / \mathrm{s})$

\begin{tabular}{cllcc}
\hline & OA PSV & OA EDV & OA mean V & OV mean V \\
\hline Control & $22.17 \pm 5.33$ & $4.10 \pm 1.66$ & $9.88 \pm 2.74$ & $(17)$ \\
$(n)$ & $(18)$ & $(18)$ & $10.56 \pm 2.94$ & 0.31 \\
NDR & $24.27 \pm 6.02$ & $4.19 \pm 1.69$ & $(22)$ & $(30)$ \\
$(n)$ & $(33)$ & $(33)$ & $8.52 \pm 2.35$ & $2.36 \pm 0.30$ \\
BDR & $21.40 \pm 4.80$ & $2.62 \pm 1.25$ & $(27) \dagger$ & $(27)$ \\
$(n)$ & $(27)$ & $(27)^{*} \#$ & 0.15 \\
\hline
\end{tabular}

OA PSV, ophthalmic artery peak systolic velocity; OA EDV, ophthalmic artery end-diastolic velocity; OA mean V, ophthalmic artery mean velocity; OV mean $\mathrm{V}$, ophthalmic vein mean velocity; NDR, non-diabetic retinopathy; BDR, background diabetic retinopathy. * Statistically significant difference from the NDR grouip $(p=0.0002)$.

\#Statistically significant difference from the control group $(p=0.003)$.

$\uparrow$ Statistically significant difference from the NDR group $(p=0.01)$ Kruskal-Wallis. 
diameter data that are needed to estimate total blood flow, we can not discuss with certainty the similarity of the pulsatile blood flow changes and our results.

Previous studies using CDI in supine diabetic patients did not find any significant difference in posterior ciliary artery circulation in NDR and BDR patients. ${ }^{9,10}$

Mendevil ${ }^{15}$ found a significantly lower posterior ciliary artery velocity in PDR patients compared with a control group, using CDI. We believe that one of the main reasons why we could detect significant changes in the posterior ciliary artery circulation in the early stages of diabetes is the posture during the CDI measurement. It has been found that the supine posture decreases the RI and increases the EDV. ${ }^{11}$ As autoregulation and the autonomic nervous response were found to be altered in diabetic patients, ${ }^{16,17}$ the correlation of change in the ocular circulation between normal subjects and diabetics when changing the posture may not be expected to be equal. Furthermore, the erect posture has been found to give a better reproducibility. ${ }^{12}$

The RI is considered to indicate the blood vessel's compliance to changes in blood pressure. ${ }^{18}$ In our results the posterior ciliary artery RI was increased in NDR patients. This may suggest that blood vessel compliance is reduced in the choroid before the clinical manifestations of DR and that this circulatory alteration may be one of the factors in the occurrence of the DR.

The results from this study indicate that as the retinopathy progresses towards PDR, the velocity of the central retinal artery blood flow decreases (Table 4 ). The decrease in blood velocity in the central retinal artery might be due to a number of reported changes in the blood and blood vessels in diabetes, such as hypercoagulability, increased stickiness and deformability of the red blood cells, hyperviscosity, thickening of the basement membrane, atherosclerosis and blood vessel tortuosity. ${ }^{2,19-22}$

Studies investigating haemodynamics in stages before PDR, reported an increased RI in the central retinal artery in NDR patients, ${ }^{23,24}$ and decreased blood velocity in the central retinal artery in patients with $\mathrm{NDR}^{23}$ and $\mathrm{BDR}^{10}$ using CDI. These results are similar to the findings in this study, except that in this study we did not detect an increased RI in our NDR patients. The reason for that is the decrease in both the EDV and the PSV of the central retinal artery of our NDR patients (see equation 1).

Altered autoregulation has been detected in the retinal circulation of NDR patients. ${ }^{25}$ Therefore, the erect position, which would decrease the pressure in the ophthalmic artery, could be expected to reduce the PSV in the central retinal artery.

The ophthalmic artery supplies total arterial blood to the eyeball. The results of ophthalmic artery circulatory parameters of decreased mean $\mathrm{V}$ and EDV in BDR correspond to the alterations in central retinal artery and posterior ciliary artery circulation at the same stage. RI in the ophthalmic artery was higher in NDR and BDR patients compared with the control group. The results were significantly higher in the case of BDR patients.
These findings are similar to the findings of Tamaki et al. ${ }^{26}$ who found increased RI in NDR, BDR and PDR patients.

Garner and Ashton ${ }^{27}$ reported a significantly smaller mean area of the ophthalmic artery ostium in DR compared with NDR patients. Decreased vessel lumen and increased blood vessel rigidity could be responsible for the rise in resistance to flow in the ophthalmic artery.

One of the main observations in this study is the similar pattern of decreased EDV and increased RI in BDR patients in all the measured arteries (Tables 3-5,

Fig. 1). Decreased EDV and increased RI are considered to represent an increase in the peripheral vascular resistivity. ${ }^{28}$ Pathological findings found in DR and diabetes that are known to increase the peripheral vascular resistivity (capillary rarefaction, atherosclerotic changes, leucocyte adhesion, increased blood viscosity $)^{29,30}$ support this circulatory finding. The increased vascular resistivity may further compromise the ocular tissue oxygenation and nourishment and may contribute to the occurrence of neovascularisation.

The central retinal artery circulation in this study follows the above-mentioned pattern as well. However, other alterations in the central retinal artery are also observed (decreased mean V and PSV) which are not present in the other two arteries (Table 4). The retinal autoregulatory dysfunction present in diabetes as well as its vulnerability to hypoxia could be among the reasons for such a finding.

Regarding the velocity in the central retinal vein, we found it to be significantly increased in BDR compared with the NDR and control groups (Table 4). RI in the central retinal vein was also significantly higher in BDR compared with the NDR and control groups in this study (Fig. 1). An IOP pulsatility effect on the retrobulbar central retinal venous outflow was detected by Michelson et al. ${ }^{31}$ that makes a straightforward comparison between central retinal artery and central retinal vein blood flow velocity impossible.

As one-third of the diabetic population are hypertensive, patients with hypertension have also been included in this study. Despite this inclusion of hypertensive patients, there were no significant BP differences among the three groups. The syndrome of hypertension, hyperlipidaemia and hyperinsulinaemia is known to be accompanied by decreased blood flow and increased peripheral vascular resistance. ${ }^{32}$ However, increased peripheral vascular resistance is a hallmark of established hypertension. In the early stages of hypertension, a normal or even decreased peripheral resistance has been found. ${ }^{32}$ The patients from this study included subjects of advanced age, in whom sclerotic changes are likely in their blood vessels, and a number of them had established hypertension. That is probably why a hyperperfusion phase was not observed in our NDR patients.

Although we can not determine total volumetric blood flow without data on the vessel's lumen, this study's results suggest an increase in peripheral vascular resistivity that may lead to ischaemia in the ocular tissue 
in diabetes. In this context, it is interesting to note that angiotensin-converting enzyme inhibitors, known to reduce the leakage in early diabetic retinopathy, ${ }^{33}$ are found to increase the EDV in the central retinal and posterior ciliary arteries of hypertensive patients ${ }^{34}$ and consequently reduce the peripheral resistivity to flow. Therefore, one of the possible targets for diabetic retinopathy management could be a therapy that would reduce the peripheral resistivity to flow, especially in the choroidal circulation, which supplies the vast ocular blood perfusion.

In conclusion, in this study we found posterior ciliary artery circulatory alterations in NDR and BDR patients suggesting choroidal circulatory dysfunction. The increased posterior ciliary artery RI in NDR patients suggests reduced vessel wall compliance in the choroid that is present before the clinical manifestations of diabetic retinopathy. A similar pattern of circulatory changes was detected in all three measured arteries (central retinal, posterior ciliary and ophthalmic artery) comprising decreased EDV and increased RI in BDR patients, which indicates an increase in ocular peripheral vascular resistivity.

\section{References}

1. Bek T. Transretinal histopathological changes in capillaryfree areas of diabetic retinopathy. Acta Ophthalmol 1994;72:409-15.

2. Yanoff M. Ocular pathology of diabetes mellitus. Am J Ophthalmol 1969;67:21-38.

3. Hidayat AA, Fine B. Diabetic choroidopathy. Ophthalmology 1985;92:512-22.

4. Geyer O, Neudorfer M, Snir T, Goldstein M, Rock T, Silver DM, Bartov E. Pulsatile ocular blood flow in diabetic retinopathy. Acta Ophthalmol Scand 1999;77:522-5.

5. MacKinnon JR, O'Brien C, Swa K, Aspinall P, Butt Z, Cameron D. Pulsatile ocular blood flow in untreated diabetic retinopathy. Acta Ophthalmol Scand 1997;75:661-4.

6. Schmidt KG, von Ruckmann A, Kemkes-Metthes B, Hammes HP. Ocular pulse amplitude in diabetes mellitus. $\mathrm{Br} \mathrm{J}$ Ophthalmol 2000;84:1282-4.

7. Langham ME, Grebe R, Hopkins S, Marcus S, Sebag M. Choroidal blood flow in diabetic retinopathy. Exp Eye Res 1991;52:167-73.

8. Schmetterer L, Salomon A, Rheinberger A, Unfried C, Lexer $\mathrm{F}$, Wolzt M. Fundus pulsation measurements in diabetic retinopathy. Graefes Arch Clin Exp Ophthalmol 1997;235:283-7.

9. Guven D, Ozdemir H, Hasanreisoglu B. Hemodynamic alterations in diabetic retinopathy. Ophthalmology 1996;103:1245-9.

10. Goebel W, Lieb WE, Ho A, Sergott RC, Farhoumand R, Grehn F. Color Doppler imaging: a new technique to assess orbital blood flow in patients with diabetic retinopathy. Invest Ophthalmol Vis Sci 1995;36:860-70.

11. Evans DW, Harris A, Garrett M, Chung HS, Kagemann L. Glaucoma patients demonstrate faulty autoregulation of ocular blood flow during posture change. Br J Ophthalmol 1999;83:809-13.

12. Hasegawa S, Nagahara M, Araie M. Color Doppler imaging in sitting patients. The 52nd Japanese Clinical Ophthalmology Congress 1998. Congress program, 259.
13. D'Amore PA. Mechanisms of retinal and choroidal neovascularization. Invest Ophthamol Vis Sci 1994;35:3974-9.

14. Hansen N, Stonestreet B, Rozenkrantz T. Validity of Doppler measurements of anterior cerebral artery blood flow velocity: correlation with brain blood flow in piglets. Pediatrics 1983;72:526-31.

15. Mendevil A. Ocular blood flow velocities in patients with proliferative diabetic retinopathy after panretinal photocoagulation. Surv Ophthalmol 1997;42:S89-95.

16. Rassam SMB, Patel V, Kohner EM. The effect of experimental hypertension on retinal vascular autoregulation in humans: a mechanism for the progression of diabetic retinopathy. Exp Physiol 1995;80:53-68.

17. Fallon TJ, Maxwell DL, Kohner EM. Autoregulation of retinal blood flow in diabetic retinopathy measured by the blue light entopic technique. Ophthalmology 1987;94:1410-5.

18. Legarth J, Nolsoe C. Doppler blood flow velocity waveforms and relation to peripheral resistance in the brachial artery. J Ultrasound Med 1990;9:449-53.

19. Chung TW, Liu AG, Yu JJH. Rheological parameter alterations in blood may impair oxygen transported to the retina in type 2 diabetes. Proceedings of the National Science Council, ROC 1994;18:30-5.

20. Bek T. Venous loops and reduplications in diabetic retinopathy: prevalence, distribution, and pattern of development. Acta Ophthalmol Scand 1999;77:130-4.

21. Ashton N. Vascular basement membrane changes in diabetic retinopathy. Br J Ophthalmol 1974;58:344-63.

22. Van Bijsterveld OP. Diabetic retinopathy. 1st ed. London: Martin Dunitz, 2000.

23. Kawagishi T, Nishizawa Y, Emoto M, Konishi T, Maekawa K, Hagiwara S, et al. Impaired retinal artery blood flow in IDDM patients before clinical manifestation of diabetic retinopathy. Diabetes Care 1995;18:1544-9.

24. Arai T, Numata K, Tanaka K, Kiba T, Kawasaki S, Saito T, et al. Ocular arterial flow hemodynamics in patients with diabetes mellitus. J Ultrasound Med 1998;17:675-81.

25. Kohner EM. The retinal blood flow in diabetes. Diabete Metab 1993;19:401-4.

26. Tamaki Y, Nagahara M, Yamashita H, Kikuchi M. Blood velocity in the opthalmic artery determined by color Doppler imaging in normal subjects and diabetics. Jpn J Ophthalmol 1993;37:385-92.

27. Garner A, Ashton N. Ophthalmic artery stenosis and diabetic retinopathy. Trans Ophthalmol Soc UK 1972;7:101-10.

28. Friedman E, Krupsky S, Lane AM, Oak S. Friedman E, Egan $\mathrm{K}$, et al. Ocular blood flow velocity in age-related macular degeneration. Ophthalmology 1995;102:640-6.

29. Laragh JH, Brenner BM. Hypertension: pathophysiology, diagnosis and management. New York: Raven Press, 1990.

30. Harris AG, Skalak R, Hatchell DL. Leukocyte capillary plugging and network resistances increased in skeletal muscle of rats with streptozotocin-induced hyperglycemia. Int J Microcirc Clin Exp 1994;14:159-66.

31. Michelson G, Harazny J. Relationship between ocular pulse pressures and retinal vessel velocities. Ophthalmology 1997;104:664-71.

32. Lind L, Lithell H. Decreased peripheral blood flow in the pathogenesis of the metabolic syndrome comprising hypertension, hyperlipidemia, and hyperinsulinemia. Am Heart J 1993;125:1494-7.

33. Kohner EM. The renin-angiotensin system and diabetic retinopathy. Klin Wochenschr 1992;69(Suppl 29):25-7.

34. Steigerwaldt RD, Belcaro GV, Laurora G, Cesarone MR, De Sinctis MT, Incandela L. Ocular and orbital blood flow in patients with essential hypertension treated with trandolapril. Retina 1998;18:539-45. 